\title{
BIODISTANCIA EN CEMENTERIOS COLONIALES DEL CENTRO-OESTE DE ARGENTINA. UN ESTUDIO COMPARATIVO DE LA VARIACIÓN MORFOLÓGICA POSTCRANEAL
}

\author{
BIODISTANCE IN COLONIAL CEMETERIES FROM CENTRAL-WEST \\ ARGENTINA. A COMPARATIVE STUDY OF POSTCRANEAL MORPHOLOGICAL \\ VARIATION
}

\author{
Daniela A. Mansegosa ${ }^{1,2}$, P. Sebastián Giannotti ${ }^{1,2}$ y Horacio Chiavazza ${ }^{2}$
}

\begin{abstract}
En este trabajo se presentan los resultados de la variación morfológica del fémur de una población del centro-oeste de Argentina que vivió durante los siglos XVII-XVIII-XIX. Las variables del fémur analizadas en este estudio son: longitud máxima, ancho epicondilar, diámetro vertical de la cabeza, diámetro subtrocantéreo antero-posterior, diámetro subtrocantéreo medio-lateral, circunferencia al medio de la diáfisis y el índice subtrocantéreo. Estas variables se comparan por sexo, periodo cronológico y estatus socio-económico con el fin de evaluar el cambio morfológico y aportar a la comprensión de la variabilidad poblacional y del proceso de mestizaje. Además, se utilizan otras dos muestras comparativas, una prehispánica de la misma región de estudio y otra española medieval, como referencias de grupos ancestrales. Los resultados obtenidos del índice subtrocantérico permiten diferenciar entre grupos con distinta ascendencia biológica. Dichos resultados se discuten en relación con la información demográfica histórica disponible e investigaciones bioarqueológicas previas.
\end{abstract}

Palabras claves: índice subtrocantérico, mestizaje, fémur, siglos XVII-XVIII-XIX.

This paper analyzes metric femoral morphological variation among historic populations (17th, 18th, and 19th centuries). Variables analyzed in this study include maximum femoral length, epicondylar breadth, vertical head diameter, anterior-posterior subtrochanteric diameter, medial-lateral subtrochanteric diameter, midshaft circumference and the subtrochanteric index. These variables are compared by sex, chronological period, and socio-economic status to assess morphological change and contribute to understanding population variability and the process of miscegenation. In addition, two other comparative samples, one preHispanic from the same study region, and the other, medieval Spanish, are used as references for ancestral groups. The results obtained from the subtrochanteric index make it possible to differentiate between groups with different biological ancestry. These results are discussed in relation to the available historical demographic information and previous bioarchaeological investigations.

Key words: Subtrochanteric index, mixed populations, femur, 17th, 18th, and 19th centuries.

Las investigaciones de biodistancia se centran en el estudio de la variación morfológica y genética dentro y entre las poblaciones humanas del pasado, lo que permite evaluar las interacciones biológicas y culturales (Pietrusewsky 2008; Pilloud y Hefner 2016). En este sentido, los restos humanos esqueléticos y dentales proporcionan la evidencia más directa y accesible para comparar la variación cuali y cuantitativa (Larsen 1997, 2001; Chrysostomou y Thompson 2016). Su estudio resulta muy complejo dado que la variación morfológica es el resultado no solo de las características genéticas, sino que también de la influencia ambiental, cultural y sus complejas interacciones (Falconer 1986). Por lo

Consejo Nacional de Investigaciones Científicas y Técnicas (CONICET), Buenos Aires, Argentina.

2 Universidad Nacional de Cuyo, Facultad de Filosofía y Letras, Instituto de Arqueología y Etnología, Laboratorio de Arqueología Histórica y Etnohistoria, Facultad de Filosofía y Letras; Área Fundacional, Centro de Investigaciones Ruinas de San Francisco (CIRSF).alitmansegosa@gmail.com; pablosebastiangiannotti@gmail.com; hchiavazza@gmail.com 
tanto, la forma y el tamaño del cuerpo pueden reflejar tanto las diferencias genéticas como las ambientales y comportamentales (Buikstra et al. 1990).

Algunas investigaciones han desarrollado numerosos esfuerzos metodológicos por encontrar los indicadores morfológicos más confiables para definir aquellas estructuras o rasgos esqueletales que reflejen un mayor componente genético que ambiental (Liberman et al. 2001; Dunn et al. 2020). Los dientes y el cráneo son los elementos más utilizados para evaluar diferencias morfológicas que permitan discutir relaciones genéticas en las poblaciones del pasado (Ross et al. 2004; Martínez Abadías et al. 2006; Fitzgerald y Hillson 2008; Bernal et al. 2010; Luna 2015). Sin embargo, cuando éstos no están disponibles, o se encuentran en mal estado de conservación, se han propuesto como alternativas métodos métricos y no métricos basados en elementos del postcráneo (Finnegan 1978; Gibert y Gill 1990; Bass 1995). Dentro de los estudios métricos, se ha propuesto que la forma de la diáfisis proximal del fémur constituye un indicador de la ascendencia biológica. La forma subtrocantérica, obtenida de los diámetros anteroposterior y mediolateral de la región proximal de la diáfisis femoral, es reconocida como un indicador discriminante entre nativos americanos y blancos y negros americanos. Gilbert y Gill (1990), determinaron que los nativos americanos exhiben una sección anteroposterior más aplanada (platimérica), mientras que en los negros y blancos americanos la sección es más circular (eumérica). Este método propone que la variación de la forma subtrocantérica está dada por factores genéticos atribuibles a cada grupo ancestral (Gilbert y Gil 1990). En este sentido, Gill (2001) sugiere que las poblaciones de origen asiático son más platiméricas que las de ascendencia africana o europea, mientras que las poblaciones mestizas exhiben una posición intermedia. Apoyando esta idea, Miller (1995) y Lovejoy et al. (2002) afirman que las diferencias poblacionales en la forma de la diáfisis proximal aparecen en la primera infancia y, por lo tanto, es probable que se deba a una fuerte influencia del factor genético.

De manera contraria, otras investigaciones han demostrado que el dimorfismo sexual y los cambios en la subsistencia y patrones de actividad física a lo largo del tiempo tienen influencia sobre la forma subtrocantérica (Ruff et al. 1984; Ruff 1987, 1995; Rockhold 1998). En este sentido, Ruff (1995) demostró que la relación entre el ancho de la pelvis y las cargas de flexión sobre la diáfisis proximal del fémur, da como resultado formas más platiméricas en las mujeres que en los hombres. De igual modo, algunos autores han demostrado una asociación entre la estrategia de subsistencia y la forma subtrocantérica, por ejemplo, en las sociedades agrícolas se ha hallado una forma más eumérica, principalmente entre los individuos masculinos, mientras que en las sociedades cazadoras recolectoras predomina una forma platimérica (Ruff et al. 1984). Esto está reforzado por evidencia experimental, clínica y antropológica que demuestra que el tamaño y la forma diafisiaria refleja las cargas mecánicas sufridas por el hueso durante la vida (Curry 1984; Ruff 1999; Frost 2003). Por consiguiente, es importante tener en cuenta la influencia de los factores ambientales y la plasticidad del esqueleto frente a los mismos.

A partir de estas dos posibilidades interpretativas, investigaciones posteriores retomaron la técnica de Gilbert y Gill (1990) para ponerla a prueba en nuevas colecciones procedentes de otras regiones geográficas y así evaluar su eficacia. Dichos estudios demostraron que los nativos americanos tienen una sección más platimérica que otras poblaciones del mundo (Gill y Rin 1990; Wescott 2001, 2005; Tallman y Winburn 2015). Otros estudios sugieren que la discriminación entre grupos con diferente ascendencia se puede lograr con mayor eficacia si adicionamos otras variables como el tamaño de la cabeza del fémur y la longitud total (Wescott 2005). En consecuencia, se considera que este método simple resulta una aproximación para estimar la ascendencia donde la clasificación del esqueleto se restringe generalmente a uno de estos tres grupos.

En Argentina, existen escasos antecedentes en el estudio métrico de los huesos postcraneales. Algunas investigaciones se han centrado en la creación de funciones discriminantes para la evaluación del sexo (Béguelin y González 2008; Béguelin et al. 2011; Mazza y Béguelin 2013; Mansegosa et al. 2018). Mientras que otras se han focalizado principalmente en la evaluación de la influencia del clima sobre el tamaño corporal (Béguelin y Barrientos 2006; Béguelin 2011) o de los patrones de subsistencia sobre la asimetría direccional en los miembros superiores e inferiores (Lotto y Béguelin 2014). Si bien estos trabajos presentan un importante antecedente para la región no incluyen en su estudio las variables del índice subtrocantérico del fémur, necesarias para la evaluación de la ancestría.

En poblaciones coloniales del norte de Mendoza se realizó una primera aproximación al análisis de 
la variación morfológica postcraneal en muestras procedentes de dos templos católicos (Mansegosa 2015a). Se estudió de manera preliminar el índice de platimería del fémur en relación con la estatura en una muestra de 62 individuos, lo cual permitió detectar algunas posibles tendencias a nivel cronológico y entre distintos sectores de entierro dentro de los templos (Mansegosa 2015b). A partir de la conquista española y de la fundación de la ciudad en el año 1561, las poblaciones que habitaron en el norte de Mendoza durante los siglos XVII-XVIII y XIX eran multiétnicas de origen nativo, europeo y africano (Prieto 1995). Las colecciones osteológicas derivadas de estas poblaciones han sido recuperadas en contextos arqueológicos de diferentes templos católicos. Se ha interpretado que las inhumaciones en dichos espacios sacros se hacían de manera segregada en base al estatus socioeconómico, ya que era más caro enterrarse dentro que afuera de los templos (Chiavazza 2005). Lo cual ha sido demostrado a partir del estudio histórico de los testamentos de la población local de la época (Prieto 2000 [1983]) y de la materialidad asociada a los entierros, es decir, las inhumaciones en ataúd $\mathrm{y}$ con ajuar funerario que predominan en el interior, en contraposición a los entierros directos con escaso ajuar del exterior (Chiavazza 2005). Esta interpretación del origen social y económico de los individuos ha sido considerada en numerosas investigaciones para discutir diferencias en las condiciones de salud, dieta, estrés metabólico y funcional, traumas y su impacto en los distintos sectores socioeconómicos de la población (Mansegosa 2016; Mansegosa y Giannotti 2017a; Giannotti et al. 2018; Mansegosa et al. 2018; Giannotti 2020a). Asimismo, existen algunos casos, como el del templo La Caridad, que no se adecuan a la diferenciación espacial de los entierros, ya que se ha planteado que estaba destinado a dar sepultura a un determinado tipo o grupo social, concretamente a los sectores pobres de la ciudad (Verdaguer 1931). En este trabajo se tendrá en cuenta la segregación espacial de los entierros en los templos, con la excepción del templo La Caridad, ya que todos o la mayoría de sus individuos corresponderían a un mismo sector socioeconómico.

Por lo tanto, dado que los lugares de entierro en la mayoría de los templos coloniales de Mendoza estaban estructurados de acuerdo al estatus socioeconómico, es posible suponer que hacia el interior de éstos estaban inhumadas personas con un mayor componente europeo, y hacia el exterior, nativos y esclavos africanos. Entre las expectativas planteadas podemos suponer que esta diferenciación sería más marcada durante los primeros momentos de contacto hacia el periodo Colonial temprano (S. XVII), dado el poco flujo génico entre las poblaciones. Hacia el periodo Colonial medio-tardío (Ss. XVIII-XIX), esperamos encontrar menos diferencias entre los individuos con distinto lugar de entierro ya que el flujo génico habría aumentado entre las distintas poblaciones (Mansegosa 2015a). Para evaluar dichas hipótesis, se propone como objetivo de este trabajo estudiar la variación morfológica postcraneal de las poblaciones que habitaron el centro-oeste de Argentina durante los siglos XVII-XVIII-XIX con el propósito de evaluar cambios a lo largo del tiempo entendido como proceso de mestizaje y la posible segregación espacial (estatus) con el origen poblacional. Adicionalmente, y de manera comparativa, se utilizan muestras prehispánicas de Mendoza y medievales españolas. Concretamente, se aplicará la técnica de Gilbert y Gil (1990) para clasificar entre nativos americanos y europeos/africanos, y además, se incluirán como variables adicionales otras tres medidas del fémur. Los datos postcraneales derivados de las tres muestras analizadas serán comparados entre sí, así como también se explorará la variación morfológica según la distribución temporal y espacial de los entierros de los templos coloniales del norte de Mendoza. La información obtenida permitirá aportar a una investigación más amplia que tiene como objetivo general el estudio de la variación y del proceso de mestizaje de las poblaciones del norte de Mendoza que incluyen el estudio de la morfología dental, craneal y composición genética, los cuales se encuentran actualmente en curso.

\section{Materiales y Métodos}

En este trabajo se utilizaron tres muestras. Por un lado, la muestra histórica americana que es el eje de discusión central y, que presenta diferentes periodos cronológicos y estratificación de acuerdo con el estatus. Por otro lado, dos conjuntos muestrales que se utilizan con fines comparativos, una muestra prehispánica americana y una muestra española medieval. Todos los fémures incluidos en esta investigación fueron medidos por uno de los autores de este trabajo (DAM). A continuación, se describe cada una de las muestras.

La muestra histórica americana está compuesta por fémures de 77 individuos adultos procedentes de cinco templos coloniales del Área Fundacional (Mendoza, Argentina). Esta colección fue excavada 
por el Centro de Investigaciones Ruinas de San Francisco, donde actualmente se encuentra depositada. Del total de individuos, $44(57 \%)$ corresponden a entierros hallados en posición primaria y 33 (43\%) formaban parte de entierros secundarios, que estaban mezclados debido, principalmente, a los procesos de re inhumación de la época (Chiavazza 2005). Se seleccionaron 77 fémures, los cuales se encontraban completos o fragmentados con la epífisis proximal y región subtrocantérica completa, sección imprescindible para poder medir las variables que se detallan más abajo. En el caso de los entierros primarios, se priorizó el fémur izquierdo y cuando éste no estaba, o estaba incompleto, se reemplazó por el derecho. En el caso de los entierros secundarios, todos los fémures seleccionados son de lateralidad izquierda, para evitar representar un mismo individuo dos veces. En todos los casos se dejaron de lado los fémures con patologías o fracturas premortem.

El total de la muestra histórica americana proviene de los siguientes templos católicos coloniales (Figura 1): San Francisco (RSF; $n=46$ ), La Caridad (LC; $n=$ 16), Santo Domingo (SD; $n=7$ ), San Agustín (SA; $\mathrm{n}=7$ ) y La Merced (LM; n=1) (Figura 1).

Todos los fémures pertenecen a individuos mayores de 20 años, ya que sus cóndilos, trocánteres y cabeza femoral se encontraban completamente fusionados (Sheuer y Black 2004). Ambos sexos están representados, 36 son femeninos y 41 son masculinos, esto se estableció a partir de múltiples

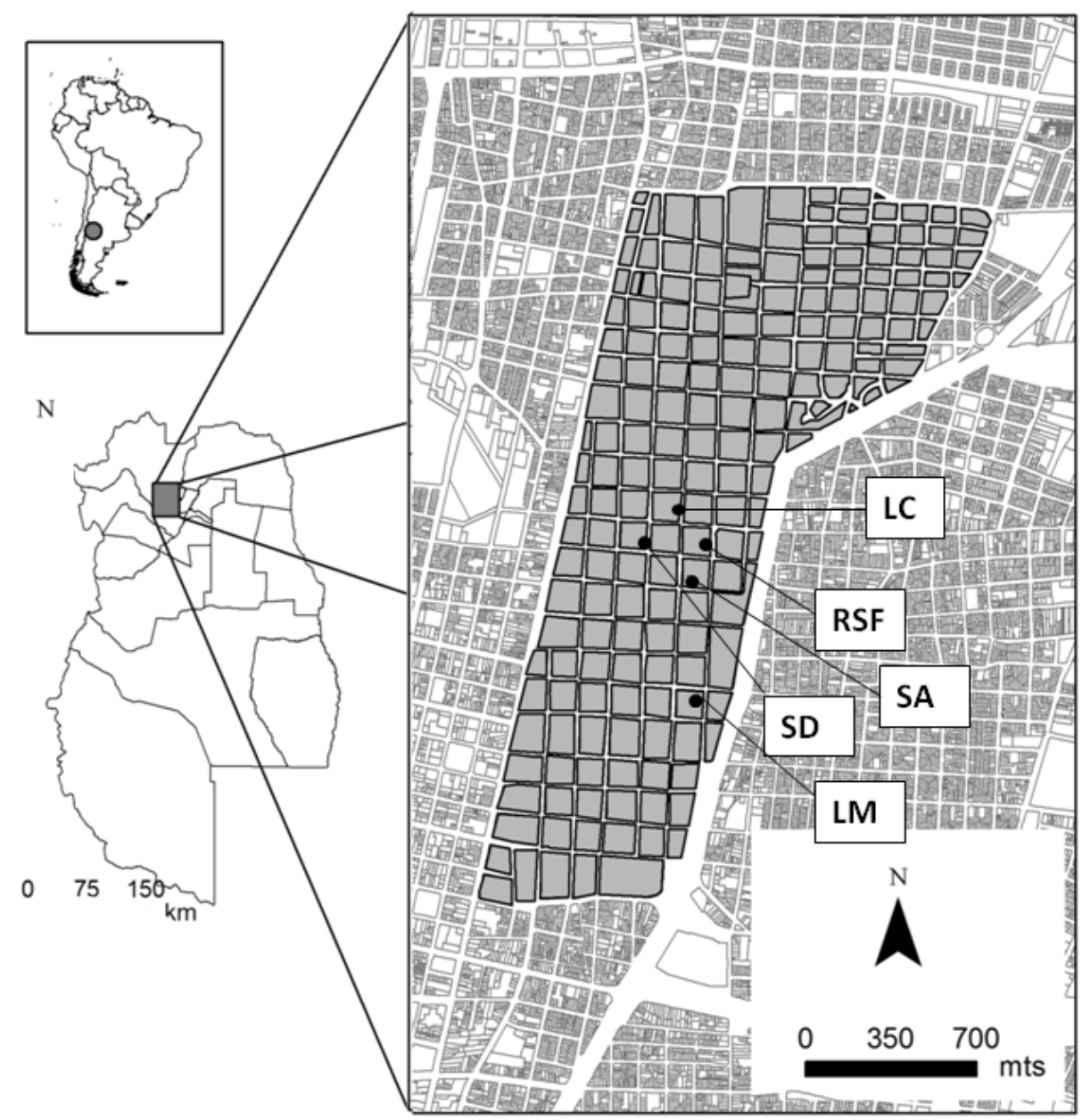

Figura 1. Ubicación de los templos coloniales pertenecientes al sitio Área Fundacional (Mendoza, Argentina). Referencias: San Francisco (RSF), La Caridad (LC), Santo Domingo (SD), San Agustín (SA) y La Merced (LM).

Location of the colonial temples belonging to the Fundacional site area (Mendoza, Argentina). References: San Francisco (RSF), La Caridad (LC), Santo Domingo (SD), San Agustín (SA) and La Merced (LM). 
métodos. En el caso de los entierros primarios se tomó en consideración las estructuras dimórficas clásicas de la pelvis y del cráneo (Buikstra y Ubelaker 1994). En los fémures aislados de los entierros secundarios se determinó el sexo mediante la aplicación de funciones discriminantes desarrolladas para estas poblaciones (Mansegosa et al. 2018) y a partir del diámetro de la cabeza femoral (Bass 1995).

Los fémures fueron agrupados según periodo cronológico y sector de entierro al cual pertenecían. Dichas clasificaciones han sido eficientemente utilizadas en estudios previos (Chiavazza 2005; Mansegosa y Giannotti 2017a). Según la agrupación cronológica los individuos fueron diferenciados en dos periodos: siglo XVII y siglos XVIII-XIX. Esta clasificación se estableció en base a asociaciones contextuales (medallas, clavazones, posicionamientos estratigráficos en relación a niveles de pisos con parches de baldosas de diferentes tipologías, y relaciones con cimientos y acumulaciones de escombros) (Chiavazza 2005 , 2008). Espacialmente fueron clasificados según sector de entierro respecto al interior o exterior del templo, los cuales representan mayor y menor estatus, respectivamente (Chiavazza 2005, 2008; Chiavazza et al. 2015a). Dado que todos los individuos inhumados en el templo LC han sido considerados previamente como "pobres y ajusticiados", en este estudio se agruparon bajo la categoría exterior, que representa a los sectores sociales de menor estatus. En la Tabla 1 se observan las características de la muestra histórica americana por templo, distribución por sexo, periodo cronológico y sector de entierro.

Por otro lado, la muestra prehispánica americana está compuesta por fémures de 36 individuos, procedentes de nueve sitios arqueológicos del norte y centro de Mendoza. Los sitios tienen diferentes cronologías que cubren un rango que va desde los
2300 a los 800 años AP. Los fémures seleccionados provienen de entierros secundarios y cumplen con las características de inclusión señaladas anteriormente en cuanto a la completitud, ausencia de patologías óseas y fracturas premortem. Las muestras se encuentran depositadas en el Museo Canals Frau de la Facultad de Filosofía y Letras (Universidad Nacional de Cuyo, Mendoza), en el Centro de Investigaciones Ruinas de San Francisco (Municipalidad de Capital de Mendoza) y en el Museo Americanista (Luján de Cuyo, Mendoza).

La muestra española medieval está compuesta por fémures de 137 individuos que fueron recuperados del Monasterio Santa María de Wamba ubicado en la provincia de Valladolid (España). La misma, corresponde a entierros secundarios del osario que fue generado a partir de reducciones de tumbas aledañas al edificio, las cuales han sido asignadas cronológicamente a los siglos XII-XVII (López-Buies 1998). La colección se encuentra depositada en el laboratorio de Antropología Física de la Facultad de Biología, de la Universidad Complutense de Madrid. Los fémures fueron medidos por la autora de este trabajo durante una estancia de capacitación en el año 2017, con la autorización del tutor Dr. Gonzalo Trancho (Tabla 2).

En ambas muestras comparativas el sexo se determinó en base a múltiples fórmulas que utilizan la altura de la cabeza del fémur (Krenzer 2006) ya que no se pudo establecer una asociación con otros elementos óseos diagnósticos del sexo.

Se midieron un total de seis variables del fémur, mediante técnicas de la morfometría tradicional (distancias lineales y perimetrales) siguiendo la metodología de Martin y Saller (1957) y las recomendaciones de Buikstra y Ubelaker (1994). Las variables registradas comprenden: longitud máxima

Tabla 1. Distribución de los fémures de muestra histórica americana según sexo, templo, periodo cronológico y estatus. Referencias de los templos: SF, San Francisco; LC, La Caridad; LM, La Merced; SD, Santo Domingo; SA, San Agustín.

Distribution of femurs in the American historical sample by sex, temple, chronological period, and status. Temple references: SF, San Francisco; LC, La Caridad; LM, La Merced; SA, San Agustín.

\begin{tabular}{lccccc|cc|cc}
\hline & \multicolumn{3}{c}{ Templo } & & & & \multicolumn{2}{c}{ Periodo cronológico } & \multicolumn{2}{c}{ Estatus } \\
& SF & LC & LM & SD & SA & XVII & XVIII-XIX & Mayor & Menor \\
\hline Masculinos & 22 & 10 & 1 & 4 & 4 & 9 & 32 & 23 & 18 \\
\hline Femeninos & 24 & 6 & 0 & 3 & 3 & 7 & 29 & 26 & 10 \\
\hline Total & 46 & 16 & 1 & 7 & 7 & 16 & 61 & 49 & 28 \\
\hline
\end{tabular}


Tabla 2. Muestras comparativas prehispánicas de Mendoza y española medieval. Sitios de procedencia, cantidad de fémures medidos, cronología y referencias.

Pre-Hispanic comparative samples, one from Mendoza, and the other, medieval Spanish. Sites of origin, number of femurs measured, chronology and references.

\begin{tabular}{lcccc}
\hline Denominación & N muestral & Cronología (años) & Procedencia & Referencias \\
\hline Los Pozos & 11 & $1700-800$ AP & Luján de Cuyo, Mendoza & Schobinger (1969-70) \\
\hline Agrelo-17 & 4 & 2300 AP & Luján de Cuyo, Mendoza & Mansegosa y Giannotti (2017b) \\
\hline Usina Sur & 5 & 1200 AP & Luján de Cuyo, Mendoza & Bárcena (1974-76) \\
\hline Potrerillos & 1 & $1500-1000$ AP & Luján de Cuyo, Mendoza & Mansegosa et al. (2017) \\
\hline $\begin{array}{l}\text { Colección } \\
\text { del Museo }\end{array}$ & 2 & $1000-500$ AP & Luján de Cuyo, Mendoza & Anzorena com.pers. (2020) \\
Americanista & 1 & $2100 \pm 90$ AP & Ciudad, Mendoza & Chiavazza (2005), Mansegosa et al. \\
\hline $\begin{array}{l}\text { Edificio Plaza } \\
\text { Huarpe }\end{array}$ & & $2100-1600$ AP & Las Heras, Mendoza & Rusconi (1962), Mansegosa et al. \\
\hline $\begin{array}{l}\text { Los Ciruelos } \\
\text { P. Godoy }\end{array}$ & 5 & 2000 AP & Godoy Cruz, Mendoza & Mansegosa y Giannotti (2019) \\
\hline $\begin{array}{l}\text { Colección } \\
\text { Semper }\end{array}$ & 5 & $1500-1000$ AP & Valle de Uco, Mendoza & Canals Frau y Semper (1956, 1957) \\
\hline $\begin{array}{l}\text { Santa María } \\
\text { de Wamba }\end{array}$ & 137 & Siglos XII-XVII & Valladolid, España & López-Bueis (1998) \\
\hline
\end{tabular}

(FLM), ancho epicondilar (FAB), diámetro vertical de la cabeza (FDC), diámetro antero- posterior subtrocantérico (FDAPS), diámetro medio-lateral subtrocantérico (FDMLS) y circunferencia al medio de la diáfisis (FCM). Las mismas se detallan en la Figura 2. Se emplearon, según la variable, calibre de corredera digital de 0,01 mm de precisión (FDC, FDAPS, FDLMS), cinta métrica (FCM) y tabla osteométrica (FLM, FAB) de $1 \mathrm{~mm}$ de precisión.

Se calculó el índice de platimería (IP) en cada uno de los fémures seleccionados según la siguiente fórmula de Krogman e Iscan (1986): IP = (FDAPS/ FDMLS*100).

La variación individual no patológica del IP varía entre 55 y 125, y se clasifica en: Platimérico (x-84,9), Eumérico $(85,0-99,9)$ y Estenomerico $(100,0-\mathrm{x})$ (Wescott 2005). Adicionalmente, se utilizó la FLM, FDC y FAB para examinar la variación del tamaño del fémur entre las muestras y complementar las interpretaciones del IP. La FLM está correlacionada con la estatura y la FCM con la masa corporal de los individuos (Trotter 1970), mientras que el FAB

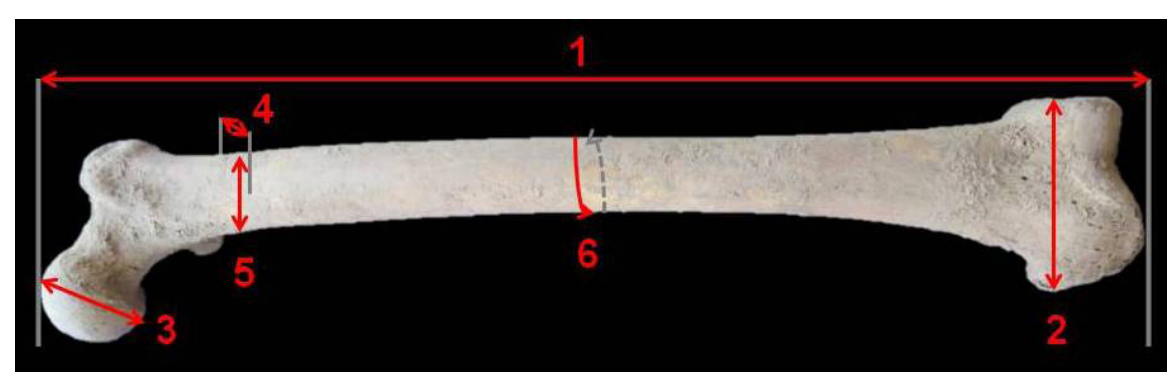

Figura 2. Detalle de las medidas realizadas en el fémur. Referencias: (1) FLM, longitud máxima; (2) FAB, ancho epicondilar; (3) FDC, diámetro vertical de la cabeza; (4) FDAPS, diámetro antero-posterior subtrocantérico; (5) FDMLS diámetro medio-lateral subtrocantérico; y (6) FCM, circunferencia al medio de la diáfisis.

Detail of measurements made on the femur. References: (1) FLM, maximum length; (2) FAB, epicondylar breadth; (3) FDC, vertical head diameter; (4) FDAPS, anterior-posterior (sagittal) subtrochanteric diameter; (5) FDMLS, medial-lateral (transverse) subtrochanteric diameter; and (6) FCM, midshaft circumference. 
se ha utilizado para evaluar el dimorfismo sexual y las diferencias poblacionales (Cavaignac et al. 2016; Gill 2001).

Se evaluó la normalidad de la distribución de las variables mediante la prueba de Kolmogorov- Smirnov. Todas las variables presentan una distribución normal. El error intraobservador fue previamente analizado seleccionando al azar un total de 40 fémures completos de la muestra histórica americana, los cuales fueron medidos en dos oportunidades, separadas por dos semanas. Las diferencias fueron evaluadas usando la prueba $\mathrm{T}$ para muestras emparejadas. En ningún caso se observaron diferencias significativas entre las mediciones, por lo que la distorsión generada por la investigadora durante el proceso de medición fue mínima (Tabla 3).

Tabla 3. Resultados de la prueba T para muestras emparejadas en el análisis de error intraobservador para medidas en el fémur.

Results of the T-test for paired samples in the intraobserver error analysis for measurements on the femur.

\begin{tabular}{lcc}
\hline \multicolumn{1}{c}{ Variables } & $t$ & $p$ \\
\hline Longitud máxima (FLM) & 0,247 & 0,808 \\
\hline Ancho epicondilar (FAB) & 2,086 & 0,056 \\
\hline Diámetro vertical de la cabeza (FDC) & $-6,97$ & 0,533 \\
\hline Diámetro antero-posterior subtrocantérico (FDAPS) & $-0,341$ & 0,741 \\
\hline Diámetro medio-lateral subtrocantérico (FDMLS) & $-0,642$ & 0,533 \\
\hline Circunferencia al medio de la diáfisis (FCM) & $-0,352$ & 0,732 \\
\hline
\end{tabular}

Para evaluar la existencia de dimorfismo sexual en los patrones de IP y variables métricas del fémur, se realizó por cada muestra una prueba $\mathrm{T}$ para muestras independientes empleando sexo (femenino, masculino) como factor independiente. Posteriormente, se clasificaron los valores arrojados por el índice de platimería en las tres categorías posibles (platimérico, eumérico y estenomérico) y se establecieron porcentajes en cada población teniendo en cuenta el sexo. Se estableció la existencia de diferencias significativas en dichos valores mediante el uso del Test de Chi cuadrado y el Test Exacto de Fisher.

Con el fin de determinar posibles diferencias poblacionales se trabajó en dos etapas. En una primera etapa más general, se compararon las tres poblaciones mediante un análisis de ANOVA de un factor utilizando sexo y muestra como factores fijos. En aquellas variables en que se determinó la existencia de diferencias entre las medias, se realizó una prueba post hoc DMS de comparaciones múltiples para determinar cuáles son aquellas medias que difieren. Además, se evaluó la existencia de diferencias significativas de los porcentajes de IP entre poblaciones mediante una prueba de Chi cuadrado y el Test Exacto de Fisher. En una segunda etapa más específica, se evaluaron diferencias morfológicas en la muestra histórica americana entre los distintos periodos cronológicos y sectores de entierro con el fin de evaluar cambios a lo largo del tiempo. Para esto se emplearon test de ANOVA de un factor en las variables cuantitativas y Chi cuadrado en las cualitativas. Todos los análisis se realizaron empleando una significancia estadística definida por una probabilidad de $p<0,05$. Para el análisis de los datos se utilizó el software estadístico SPSS 15.0.

\section{Resultados}

\section{Dimorfismo sexual}

Los resultados de las estadísticas descriptivas de cada variable, por muestra y por sexo se presentan en las Tablas 4 y 5 . En la Tabla 4 se observan los resultados de las variables cuantitativas (IP, FLM, FDC, FAB). En dicha tabla los resultados de la prueba $\mathrm{T}$ indican que las mujeres tienen una región subtrocantérica levemente más aplanada que los hombres, aunque estas diferencias no son estadísticamente significativas, por lo que se interpreta que no existen diferencias significativas entre el IP y el sexo. En cambio, sî existen diferencias entre las medidas de las variables FLM, FDC y FAB entre los sexos, con la excepción del largo del fémur y ancho epicondilar en la muestra de prehispánicos americanos.

En la Tabla 5 se observa la clasificación y distribución de los fémures de acuerdo con las categorías posibles (eumérico, platimérico y estenomérico), así como también se muestran los resultados de los test de Chi cuadrado y Exacto de Fisher, los cuales indican que no hay relación entre tipo de fémur y el sexo. De esta manera, se considera que el dimorfismo sexual no afecta el IP, y es posible evaluar diferencias poblacionales agrupando hombres y mujeres en un solo conjunto. En cambio, el resto de las medidas del fémur deben ser comparadas teniendo en cuenta el sexo.

\section{Variación entre poblaciones}

Como se presentó anteriormente en el resumen estadístico de la Tabla 4, los nativos americanos 
Tabla 4. Estadísticos descriptivos de variables cuantitativas por muestra y sexo. Valores p de las pruebas T (* y en negrita los resultados con significancia estadística). Referencias: MT, muestra total; F, femeninos; M, masculinos; I, sexo indeterminado.

Descriptive statistics of quantitative variables by sample and sex. P values of the T tests $(*$ and, in bold, the results with statistical significance). References: MT, total sample; F, female; M, male; I, indeterminate sex.

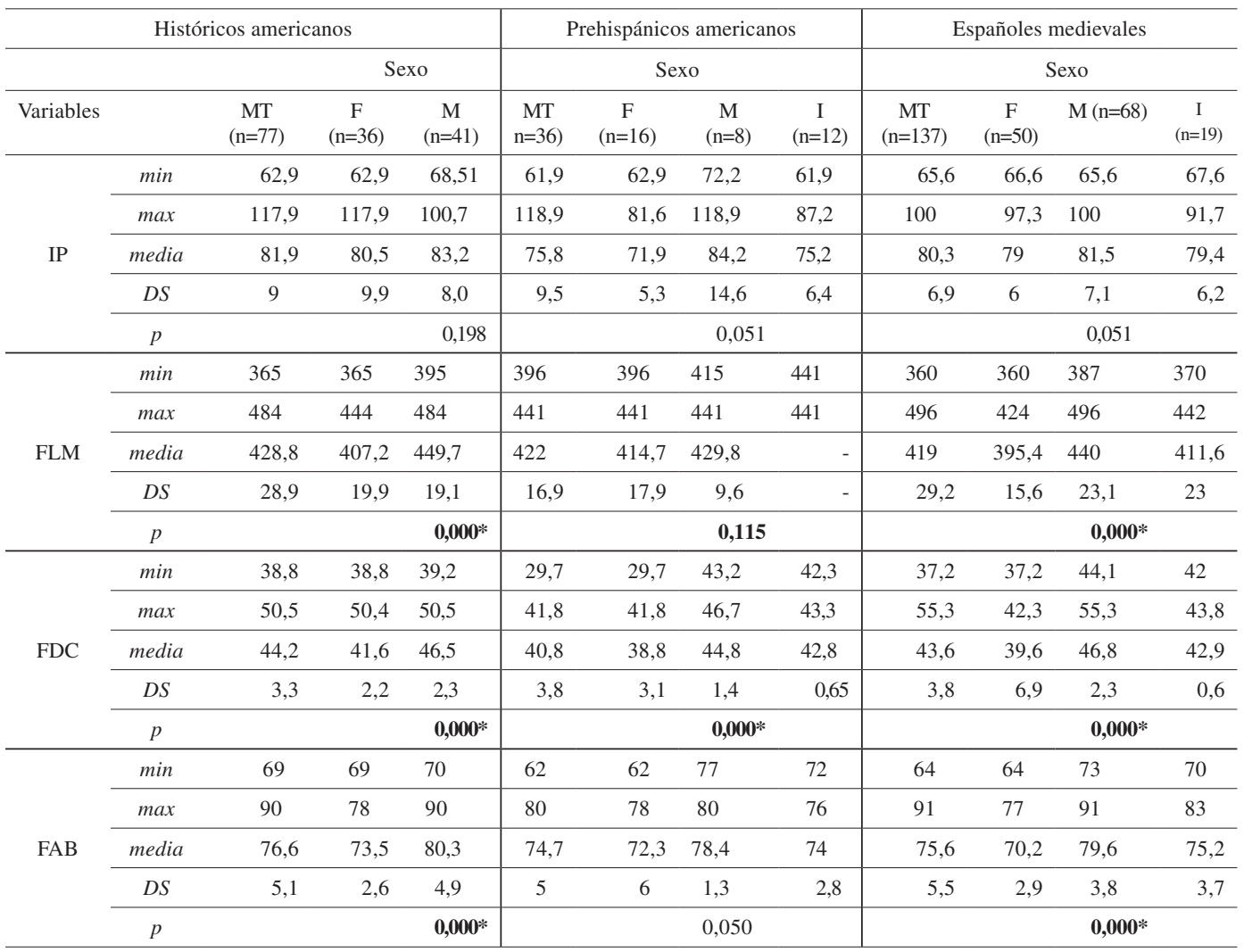

Tabla 5. Clasificación de los fémures de acuerdo con el IP, porcentajes por sexo y muestra. Valor p de las pruebas estadísticas Chi cuadrado y Exacto de Fisher. Referencias: MT, muestra total; F, femeninos; M, masculinos; I, sexo indeterminado.

Classification of the femurs according to the IP, percentages by sex and sample. P value of the statistical tests, Chi square and Fisher's Exact. References: MT, total sample; F, female; M, male; I, indeterminate sex.

\begin{tabular}{|c|c|c|c|c|c|c|c|c|c|c|c|}
\hline \multicolumn{4}{|c|}{ Históricos americanos } & \multicolumn{4}{|c|}{ Prehispánicos americanos } & \multicolumn{4}{|c|}{ Españoles medievales } \\
\hline & & \multicolumn{2}{|c|}{ Sexo } & \multicolumn{4}{|c|}{ Sexo } & \multicolumn{4}{|c|}{ Sexo } \\
\hline Variables & $\begin{array}{c}\text { MT } \\
(n=77)\end{array}$ & $\begin{array}{c}F \\
(n=36)\end{array}$ & $\begin{array}{c}M \\
(n=41)\end{array}$ & $\begin{array}{c}\text { MT } \\
(n=36)\end{array}$ & $\begin{array}{c}F \\
(n=16)\end{array}$ & $\begin{array}{c}M \\
(n=8)\end{array}$ & $\begin{array}{c}\mathrm{I} \\
(\mathrm{n}=12)\end{array}$ & $\begin{array}{c}\text { MT } \\
(n=137)\end{array}$ & $\begin{array}{c}\mathrm{F} \\
(\mathrm{n}=50)\end{array}$ & $\begin{array}{c}M \\
(n=68)\end{array}$ & $\begin{array}{c}\mathrm{I} \\
(\mathrm{n}=19)\end{array}$ \\
\hline Platimérico (\%) & 67,5 & 77,8 & 58,5 & 91,7 & 100 & 75 & 91,7 & 71,5 & 82 & 61,8 & 71,5 \\
\hline Eumérico (\%) & 28,6 & 19,4 & 36,6 & 5,6 & 0 & 12,5 & 8,3 & 27,7 & 18 & 21,1 & 27,7 \\
\hline Estenomérico(\%) & 3,9 & 2,8 & 4,9 & 2,8 & 0 & 12,5 & 0 & 0,7 & 0 & 0 & 0,7 \\
\hline$p$ & & 0,200 & & & & 0,131 & & & & 0,144 & \\
\hline
\end{tabular}

prehispánicos exhiben el IP promedio más bajo $(75,8)$, seguido por la muestra española $(80,3)$ y finalmente la histórica americana con un promedio muy similar (81,9). Estas diferencias son estadísticamente significativas (ANOVA de un factor, $\mathrm{p}=0.001$ ), siendo la media de los prehispánicos americanos la única que difiere significativamente (Tabla 6). Respecto de los porcentajes de tipo de platimería por población (Tabla 5), se observa que los prehispánicos americanos exhiben la mayor cantidad de individuos platiméricos $(91,7 \%)$, 
siendo las mujeres $100 \%$ platiméricas; la muestra española y la histórica americana tienen porcentajes más bajos, $71,5 \%$ y $67,5 \%$ respectivamente; estas diferencias resultaron significativas (Exacto de Fisher, $\mathrm{p}=0,007)$. De esta manera, se puede interpretar que desde el punto de vista de la forma subtrocantérica del fémur la muestra histórica americana y la española son más similares entre sí, y se alejan significativamente de la prehispánica americana, la cual exhibe los IP más bajos y la mayor cantidad de individuos con fémures aplanados. En la Figura 3 se observa la distribución de los individuos por muestra de acuerdo con las variables FDAPS y FDMLS que definen el IP.

Por otra parte, los resultados de la comparación de las tres poblaciones de acuerdo con las medias de las variables métricas del fémur (FDC, FLM, FAB) se presentan por sexo debido a la incidencia del dimorfismo sexual (Tabla 4). En primer lugar, entre

Tabla 6. Resultados de la comparación múltiple de medias (DMS) de las variables del fémur por sexo. *La diferencia de medias es significativa al nivel 0,05.

Results of the multiple comparison of means (MCM) of the femur variables by sex. *The mean difference is significant at the 0.05 level.

\begin{tabular}{lllcc}
\hline \multicolumn{2}{c}{ Comparación } & \multicolumn{1}{c}{ Femeninos } & Masculinos \\
\hline Variable & \multicolumn{1}{c}{ Submuestra } & \multicolumn{1}{c}{ Submuestra } & (sig.) & (sig.) \\
\hline IP & Históricos Americanos & Prehispánicos Americanos & $\mathbf{0 , 0 0 0 *}$ & \\
& Históricos Americanos & Españoles Medievales & 0,150 & \\
& Prehispánicos Americanos & Españoles Medievales & $\mathbf{0 , 0 0 3 *}$ & \\
\hline FDC & Históricos Americanos & Prehispánicos Americanos & $\mathbf{0 , 0 0 0 *}$ & 0,093 \\
& Históricos Americanos & Españoles Medievales & $\mathbf{0 , 0 0 0 *}$ & 0,584 \\
& Prehispánicos Americanos & Españoles Medievales & 0,189 & $\mathbf{0 , 0 4 2}$ \\
\hline FLM & Históricos Americanos & Prehispánicos Americanos & 0,285 & 0,064 \\
& Históricos Americanos & Españoles Medievales & $\mathbf{0 , 0 0 7 *}$ & 0,056 \\
& Prehispánicos Americanos & Españoles Medievales & $\mathbf{0 , 0 0 4 *}$ & 0,312 \\
\hline \multirow{2}{*}{ FAB } & Históricos Americanos & Prehispánicos Americanos & 0,370 & 0,341 \\
& Históricos Americanos & Españoles Medievales & $\mathbf{0 , 0 0 0 *}$ & 0,484 \\
& Prehispánicos Americanos & Españoles Medievales & 0,122 & 0,522 \\
\hline
\end{tabular}
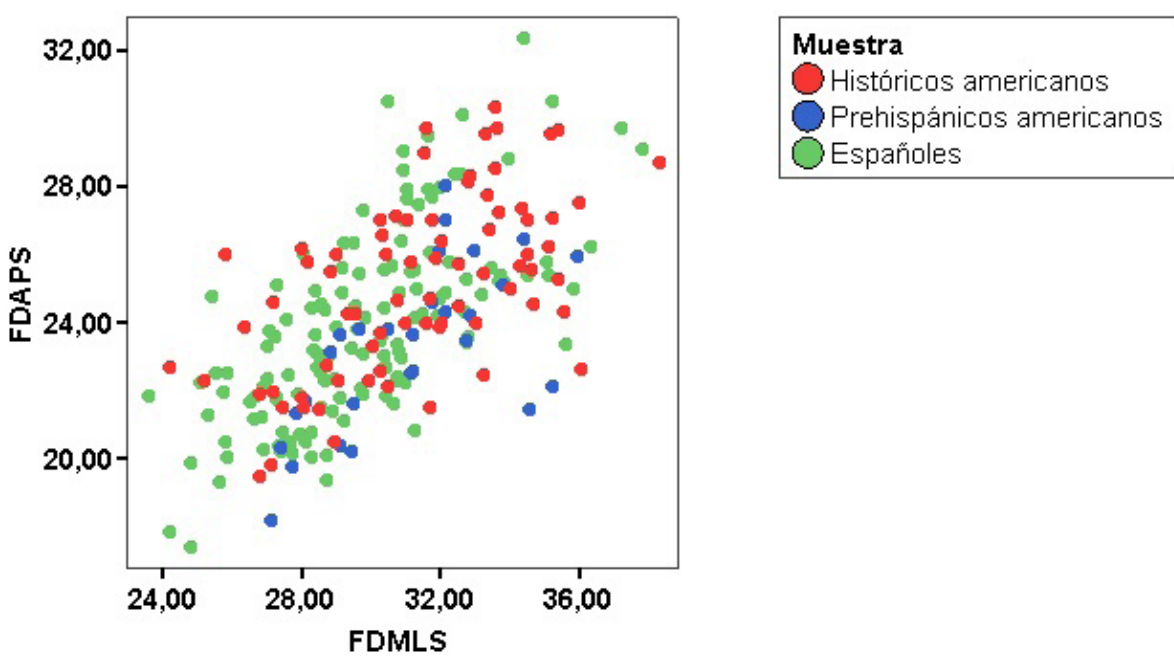

Figura 3. Distribución de los individuos según tipo de muestra de acuerdo con las variables FDAPS y FDMLS.

Distribution of individuals according to sample type together with the FDAPS and FDMLS variables. 
los individuos femeninos los análisis de ANOVA de un factor arrojaron que existen diferencias significativas entre los grupos poblacionales para las tres variables estudiadas (FDC, $\mathrm{p}=0,000$; FLM, $\mathrm{p}=0,002 ; \mathrm{FAB}, \mathrm{p}=0,000)$. Mediante los resultados del análisis post hoc DMS de comparación múltiple se pueden identificar cuáles son los conjuntos que difieren y para qué variables, estos resultados se muestran en la Tabla 6. En todas las variables, la muestra histórica americana de fémures femeninos se diferencia significativamente de la española. En segundo lugar, entre los individuos masculinos, hay mayor solapamiento de las medidas, el análisis de ANOVA de un factor indica que solo hay diferencias significativas en las medidas de la variable FDC, concretamente, entre individuos prehispánicos americanos y españoles $(p=0,042)$.

\section{Variación por periodo cronológico y estatus en los individuos históricos americanos}

El resultado del ANOVA de un factor para el IP indica que no existen diferencias significativas en los promedios entre los distintos grupos cronológicos y de estatus de la muestra histórica americana $(\mathrm{p}=0,310)$, lo cual puede deberse al tamaño pequeño de la muestra. Sin embargo, se observa que el IP promedio de los grupos de bajo estatus de ambos periodos cronológicos es menor que el hallado en los grupos de mayor estatus de los dos periodos (Tabla 7). Los porcentajes de cantidad de individuos platiméricos son mayores entre los individuos de menor estatus. En contraste, resulta interesante que los tres individuos (100\%) del siglo XVII de mayor estatus sean euméricos. Sin embargo, estas diferencias no resultan significativas desde el punto de vista estadístico (Exacto de Fisher, $\mathrm{p}=3$,24) (Tabla 8).

En los fémures femeninos las variables FML y FAB no mostraron diferencias (ANOVA de un factor, $\mathrm{p}=0,919$ y $\mathrm{p}=0,256$ respectivamente), sin embargo, la variable FDC sí muestra que existen diferencias significativas (ANOVA de un factor, $\mathrm{p}=0,48$ ). Desafortunadamente la muestra es tan pequeña que no se pudieron realizar pruebas post hoc para ver cuáles son las medidas que difieren. En los masculinos pasa algo similar ya que el ANOVA de un factor indica que no hay diferencias significativas en las variables FML ( $\mathrm{p}=0,571)$ y FAB $(\mathrm{p}=0,796)$, mientras que sí lo son en la variable FDC $(\mathrm{p}=0,43)$. La prueba post hoc indica que se trata específicamente de las muestras de bajo y alto estatus de los siglos XVIII-XIX, que tienen una media de 50,37 y 45,86 respectivamente. Es decir que los individuos masculinos de menor estatus del periodo XVIII-XIX presentan la cabeza femoral más robusta que sus contemporáneos de mayor estatus.

Tabla 7. IP en la muestra histórica americana subdividida por grupo cronológico y estatus.

IP in the American historical sample subdivided by chronological group and status.

\begin{tabular}{lcccc}
\hline \multicolumn{1}{c}{ Cronología y estatus } & Media & DS & Mínimo & Máximo \\
\hline XVII- estatus bajo $(\mathrm{n}=6)$ & 81,40 & 5,32409 & 74,86 & 88,77 \\
\hline XVII - estatus alto $(\mathrm{n}=3)$ & 89,12 & 4,41891 & 86,18 & 94,20 \\
\hline XVIII XIX - estatus bajo $(\mathrm{n}=12)$ & 80,58 & 7,15592 & 72,73 & 93,57 \\
\hline XVIII XIX- estatus alto $(\mathrm{n}=20)$ & 84,39 & 9,18254 & 68,51 & 100,70 \\
\hline
\end{tabular}

Tabla 8. Clasificación según tipo de platimería y porcentaje por grupo cronológico y estatus en la muestra histórica americana.

Classification according to platimery type and percentage by chronological group and status in the American historical sample.

\begin{tabular}{lcccc}
\hline & $\begin{array}{c}\text { XVII-bajo } \\
(\mathrm{n}=6)\end{array}$ & $\begin{array}{c}\text { XVII-alto } \\
(\mathrm{n}=3)\end{array}$ & $\begin{array}{c}\text { XVIII XIX-bajo } \\
(\mathrm{n}=12)\end{array}$ & $\begin{array}{c}\text { XVIII XIX-alto } \\
(\mathrm{n}=20)\end{array}$ \\
\hline Platimérico $(\%)$ & 66,7 & 0,0 & 66,7 & 60,0 \\
\hline Eumérico $(\%)$ & 33,3 & 100,0 & 33,3 & 30,0 \\
\hline Estenoméricos $(\%)$ & 0,0 & 0,0 & 0,0 & 10,0 \\
\hline
\end{tabular}




\section{Discusión}

El objetivo de este trabajo fue estudiar la variación morfológica postcraneal de las poblaciones que habitaron el centro-oeste de Argentina durante los siglos XVII-XVIII-XIX para evaluar, por un lado, cambios a lo largo del tiempo y por otro, la relación entre estatus socio-económico y el origen poblacional. Adicionalmente, para tener referencias de grupos ancestrales se estudiaron otras dos muestras con fines comparativos, una de origen prehispánico americano y la otra medieval española. De esta manera, la investigación se orientó a comparar poblaciones de distinto origen que puedan aportar a la comprensión del proceso de mestizaje de poblaciones multiétnicas como las coloniales americanas. La morfología del fémur, en particular la forma de subtrocantérica ha sido considerada un indicador de la ancestría poblacional, aunque también puede reflejar diferencias ambientales o de uso del cuerpo que influyen en la plasticidad del esqueleto (Ruff 1999; Gill 2001; Frost 2003). En términos generales, los resultados hallados en este trabajo indican que la forma subtrocantérica (IP) permitió hallar diferencias significativas entre poblaciones. A su vez, la población americana histórica de la época colonial, manifestó diferencias importantes entre los siglos, así como también, entre individuos pertenecientes a distintos estatus socio-económico. Sin embargo, las otras medidas examinadas en el fémur, como la longitud total, el ancho epicondilar y el diámetro vertical de la cabeza arrojaron información variable más difícil de interpretar.

\section{Morfología del fémur y el dimorfismo sexual}

Las mujeres de las tres muestras analizadas en este trabajo resultaron más platiméricas que los hombres, aunque esta diferencia no es estadísticamente significativa. Esta tendencia, de una mayor platimería en mujeres respecto de los hombres, también ha sido registrada en poblaciones hispanas, polinesias, nativas de Norteamérica y en poblaciones blancas americanas modernas (Wescott 2005). Siguiendo la propuesta de Ruff (1987), la forma más aplanada de los fémures femeninos puede ser resultado de factores biomecánicos propios del sexo. Esto es así ya que la mayor amplitud pélvica (vinculada al embarazo y al parto) y, en consecuencia, la mayor distancia interacetabular produce un incremento de la flexión mediolateral que da como resultado la forma más platimérica del fémur (Ruff 1987).
Las otras variables estudiadas, indican que las mujeres tienen fémures más pequeños que los hombres, tanto en su largo, como en el ancho epicondilar y el diámetro de la cabeza, por lo que el dimorfismo sexual sí estaría influyendo de manera significativa en el tamaño femoral. Esto también ha sido ampliamente documentado en otras poblaciones, e inclusive son variables que se utilizan para la creación de funciones discriminantes para determinar el sexo (Caiaffo et al. 2019; Cavaignac et al. 2016; Krenzer 2006).

Por lo tanto, dado que el dimorfismo no influyó en la forma subtrocantérica del fémur, el IP es una buena variable para comparar conjuntos mixtos permitiendo una estadística más robusta en términos de tamaño de las muestras. En cambio, las otras tres variables (FLM, FDC y FAB), sí presentan un patrón dimórfico diferencial, lo cual llevó a tener que realizar comparaciones separadas entre los sexos, con tamaños muestrales más pequeños.

\section{Morfología del fémur, grupos ancestrales y diferencias entre poblaciones}

La muestra histórica americana se diferenció de la prehispánica y de la española, exhibiendo una sección subtrocantérica del fémur más redondeada. La muestra prehispánica americana registraron fémures más aplanados, que tienen su mayor expresión entre los individuos femeninos, lo cual coincide con lo documentado en poblaciones nativas americanas de otras regiones (Gill y Rin 1990; Wescott 2001, 2005). En cambio, las muestras españolas tienen IP más bajo de lo esperado (Wescott y Srikanta 2008), lo cual puede relacionarse con factores vinculados con la variación genética, diversidad ambiental o factores biomécánicos del uso del cuerpo (Ruff et al. 1984).

En las tres poblaciones se registraron los tres tipos de platimería (platimérico, eumérico, estenomérico), aunque en general, presentan un mayor porcentaje de individuos platiméricos. La muestra de nativos americanos es la que tiene el mayor porcentaje de individuos con fémures aplanados $(91,7 \%)$, lo cual es lo esperado. En cambio, la población histórica americana y la medieval española, tienen menor cantidad de platiméricos y registran más euméricos, aunque en porcentajes más bajos que los valores de referencia de otras poblaciones blancas americanas y españolas, donde los porcentajes de individuos euméricos informados son mayores (Wescott 2005, 2006; Wescott y Zephro 2016). Esto hace pensar que la población histórica americana tiene una mayor 
variabilidad genética, y probablemente un mayor componente amerindio.

Por otra parte, el tamaño del fémur refleja diferencias menos claras entre las poblaciones. Cuando evaluamos a los individuos femeninos, las diferencias del tamaño del fémur (FLM, FAB, FDC) solo son significativas entre mujeres americanas históricas y españolas, siendo más grandes los fémures de las primeras que de las segundas. Interesa destacar que, si bien las diferencias no son tan marcadas, las mujeres prehispánicas tienen fémures más grandes que las de las otras dos poblaciones. Probablemente, el hecho de que las mujeres americanas coloniales exhiban fémures de tamaños intermedios se deba al proceso de mestizaje, lo cual acompaña las tendencias del IP. Complementariamente, entendemos que el tamaño del fémur como indicador de la estatura, está influenciado por factores ambientales tales como la dieta y actividad física. En este sentido las poblaciones americanas prehispánicas e históricas, se diferencian tanto en la dieta como en los patrones de actividad física asociados a la subsistencia, debido al cambio de estilo de vida a partir de la conquista española hacia un mayor sedentarismo y aumento del consumo de alimentos agrícolas (Chiavazza et al. 2015b). En el marco de esta hipótesis, creemos que la actividad física influye en las diferencias si se considera que las muestras proceden de sociedades con estilos de vida notoriamente distintos. En este sentido, el ancho epicondilar (FAB), además de ser un indicador de dimorfismo sexual, puede informar acerca de la actividad física al ser un área próxima a la metáfisis distal. La misma está sujeta a mayores tensiones de flexión ante la locomoción a altas velocidades (i.e. correr) por el comportamiento de los músculos situados alrededor (cuádriceps, isquiotibiales y gastrocnemio) (Novacheck 1998). Por ende, es posible sugerir que la diferencia significativa del FAB entre la muestra histórica y española se explica por el dimorfismo sexual (menor dimorfismo en alguna de las dos muestras) y/o por la actividad física (locomoción). Por otro lado, el diámetro máximo de la cabeza (FDC), empleado fundamentalmente para estimar la masa corporal de un individuo (Ruff y Auerbach 2004), sugiere diferencias significativas en el peso corporal entre las mujeres de la muestra histórica y prehispánica y entre históricos y españoles. Es importante destacar que estudios de estrés funcional previos en entierros primarios de la muestra histórica evaluado entre sexos hallaron bajas diferencias significativas (para cambios entésicos y enfermedad degenerativa articular) o nulas (para índices pilástrico, platimérico y cnémico) (Giannotti 2020a, 2020b). En definitiva, las diferencias detectadas no pueden ser explicadas exclusivamente por la actividad física (la cual pareciera ser relativamente baja) y la discusión acerca de la variación biológica de acuerdo a distintos patrones ancestrales cobra mayor sustento.

En cuanto a los fémures masculinos, éstos muestran un mayor solapamiento en las medidas de FLM y FAB entre las poblaciones. Solo los prehispánicos y españoles se diferencian únicamente en la medida de FDC. En términos generales los fémures masculinos de la muestra histórica americana son de mayor tamaño que las otras dos muestras, esta tendencia sigue a la observada en los individuos femeninos.

\section{Variabilidad morfológica dentro de la población colonial americana, mestizaje y demografía}

Las muestras históricas americanas en su conjunto muestran un IP que va desde 67,5 a 117,9, con un promedio de 81,9 , lo cual da cuenta de una gran variabilidad interna. Cuando se clasifican en términos cualitativos el $67,5 \%$ resulta de tipo platimérico, mientras que el $28,6 \%$ cae dentro del rango eumérico y solo el $3,9 \%$ es estenomérico. Esta variabilidad puede ser interpretada como resultado de la agregación y flujo génico de poblaciones extra-regionales europeas, africanas y hasta grupos nativos no locales como bien ha sido documentado por la historia (Prieto 2000 [1983]). Cuando subdividimos la muestra por cronología y estatus se observa que hacia el siglo XVII las muestras de mayor estatus tienen fémures con una sección más redondeada ( $\mathrm{IP}=89,2)$ que aquellos de menor estatus que resulta más aplanada $(\mathrm{IP}=81,4)$, es decir que hay marcada diferenciación entre los conjuntos. Los fémures redondeados en los individuos de mayor estatus y de los primeros momentos de contacto, dan cuenta de la presencia europea en la región con un escaso flujo génico amerindio (100\% de los individuos del interior son euméricos). Existen registros históricos que permiten complementar la información obtenida. Para principio del siglo XVII se contabilizan entre 200 o 250 pobladores españoles, de los cuales el $70 \%$ eran hombres. Y hacia fines de siglo esta cifra se duplica (Zuluaga 1964). De esta manera, interpretamos que tales pobladores europeos han sido inhumados en el interior de los templos, dado la morfología eumérica de los fémures analizados. Asimismo, vemos la coexistencia con la población nativa en este mismo periodo a través de los individuos con fémur platimérico enterrados 
fuera de los templos (66,7\%). En este último punto, aclaramos que también hay fémures euméricos en el exterior $(33,3 \%)$, dado que era un sector de menor estatus nos inclinamos a pensar que puede tratarse de individuos de ascendencia africana y no europea. La presencia africana para este periodo Colonial está documentada por la historia, ya que se menciona que el 25\% de la población de la ciudad de Mendoza eran esclavos ((Prieto 2000 [1983]).

Posteriormente, hacia los siglos XVIII-XIX, la tendencia morfológica registrada en los fémures se mantiene, aunque el IP disminuye en los individuos de mayor estatus ( $\mathrm{IP}=84,39 ; 30 \%$ euméricos). Es decir que los fémures de los individuos del interior se vuelven un poco más aplanados a lo largo del tiempo. Lo cual podría estar relacionado con el aumento del mestizaje y la incorporación de genes amerindios en las familias europeas. En este sentido, la historia menciona que el desequilibrio demográfico entre los sexos dentro de los europeos favoreció el flujo génico entre poblaciones a pesar de las normas maritales de la época que prohibían la mezcla de sangre (Prieto 1995). Sin embargo, dicha visión romántica del mestizaje difundida por la historia tradicional, ha sido desmitificada en numerosos trabajos, donde se denuncia que las violaciones por parte de europeos a mujeres y niñas indígenas era una práctica ampliamente ejecutada (Blanchard et al. 2018), lo que sin duda resultó en mestizajes dentro de los grupos nativos de la región. Los estudios demográficos de Zuluaga (1970) mencionan que los habitantes de la ciudad para mediados de siglo XVIII alcanzan unos 4000 habitantes, de los cuales solo unos 150 eran españoles masculinos con bienes. El resto de los habitantes eran españoles y criollos españolizados del estrato hispano-criollo bajo, indios, esclavos africanos y mulatos (Prieto 2000 [1983]). Esta información da cuenta de las fuertes interacciones poblacionales hacia finales del periodo Colonial, que se ven reflejadas en el postcráneo a partir del cambio de la forma subtrocantérica del fémur.

Respecto del tamaño del fémur, en la muestra histórica americana, no se registraron diferencias marcadas en la longitud y ancho epicondilar, con la excepción de los individuos masculinos de menor estatus del periodo XVIII-XIX que presentan la cabeza femoral más robusta que sus contemporáneos de mayor estatus. Siendo el tamaño del fémur una medida relacionada con el tamaño corporal (Trotter 1970), podemos interpretar que no habría diferencias marcadas en la estatura entre los distintos grupos poblacionales, las cuales se volvían mucho más homogéneas a lo largo del tiempo.

En términos generales, los resultados de cambios morfológicos a lo largo del tiempo y variación morfológica que se corresponde con la segregación espacial en los sectores de entierro se complementan con los indicadores de salud encontrados en investigaciones anteriores. La presencia de hipoplasias de esmalte dental e hiperostosis porótica es mayor entre las personas enterradas en los sectores de menor estatus, lo que indica perores condiciones de salud (Mansegosa 2015, 2016; Mansegosa et al. 2018). Estilos de vida diferentes entre estos sectores poblacionales se han detectado en los patrones de desgaste dental diferentes y la presencia de caries, que han sido interpretados como diferencias en el procesamiento diferencial de los alimentos. Las personas de mayor estatus consumían alimentos más blandos y cariogénicos que aquellos de sectores de menores recursos (Giannotti et al. 2018; Mansegosa 2016). De esta manera, los indicadores morfológicos entre grupos de estatus socioeconómico indican que estas personas pertenecían a grupos ancestrales diferentes: mayor componente europeo entre los individuos de mayor estatus y un mayor componente indígena den el grupo de menores condiciones socio-económicas. Sin embargo, a lo largo del tiempo estas diferencias morfológicas se ven homogeneizadas por el proceso de mestizaje y el flujo génico.

\section{Conclusiones}

Las muestras históricas americanas analizadas en este trabajo mostraron una diferenciación de la forma de la región subtrocantérica del fémur que las diferenció de las prehispánicas americanas y de las medievales europeas. Cuando se analizó la variación morfológica por periodo cronológico y estatus socio-económico se observó que los individuos de mayor estatus, es decir aquellos inhumados en los sectores internos de los templos exhibían un fémur más redondeado que lo acerca a lo esperado para poblaciones de origen europeo. En cambio, los individuos procedentes de poblaciones de menores recursos socioeconómicos, es decir enterrados en los sectores externos de los templos, exhiben un fémur más aplanado indicando una ancestría amerindia. Esta diferenciación es más marcada en el siglo XVII y luego comienza a hacerse menos fuerte. Es decir, aumenta la variabilidad hacia el interior de los sectores de entierro (grupo de estatus) en los siglos 
XVIII-XIX, indicando una mayor homogeneidad debido al flujo génico entre poblaciones.

En un futuro se podrán vincular estos resultados con los obtenidos de ADN antiguo que se encuentran en proceso de análisis (Cardozo et al. 2019), así como también con otros estudios morfométricos derivados de la antropología dental, lo cual ayudará a poner a prueba dichas interpretaciones y profundizar nuestra comprensión de las características y forma de vida de las poblaciones coloniales que habitaron el centro-oeste argentino.

Agradecimientos: A los miembros del Centro de Investigaciones Ruinas de San Francisco
(Municipalidad de Mendoza), que colaboraron con las tareas de excavación de las muestras históricas americanas estudiadas en este trabajo. Al Ministerio de Cultura de la Nación (República Argentina), quien otorgó una beca a DAM para realizar una estancia de capacitación en la Facultad de Biología de la Universidad Complutense de Madrid, donde se encuentra depositada la muestra española medieval aquí estudiada. Al Dr. Gonzalo Trancho por su guía y trabajo colaborativo durante la estancia de capacitación. Destacamos los aportes de los cuatro evaluadores anónimos, quienes ayudaron a mejorar notablemente la versión previa de este texto.

\section{Referencias Citadas}

Bárcena, J. 1974-76. Antropología física del Valle de Uspallata. Provincia de Mendoza. Anales de Arqueología y Etnología 29-31:109-184.

Bass, W. 1995. Human osteology. A laboratory and field manual. Special Publication $N^{\circ}$ 2. Missouri Archaeological Society, Columbia.

Béguelin, M. 2011. Variación Geográfica en la Morfología del Esqueleto Postcraneal de las Poblaciones Humanas de Pampa y Patagonia durante el Holoceno Tardío: Una Aproximación Morfométrica. British Archaeological Reports, International S2253, Oxford.

Béguelin, M., F. Lotto y P. González 2011. Estimación del sexo en cazadores-recolectores de Sudamérica a partir de variables métricas del húmero. Intersecciones en Antropología 12:61-68. Béguelin, M. y G. Barrientos 2006. Variación morfométrica postcraneal en muestras tardías de restos humanos de Patagonia: una aproximación biogeográfica. Intersecciones en Antropología 7:49-62.

Béguelin, M. y P. González 2008. Estimación del sexo en poblaciones del sur de Sudamérica mediante funciones discriminantes para el fémur. Revista Argentina de Antropología Biológica 10:55-70.

Bernal, V., S.I. Perez, P. Gonzalez, M. Sardi y H.M. Pucciarelli 2010. Spatial patterns and evolutionary processes in southern south America: a study of dental morphometric variation. American Journal of Physical Anthropology 142:95-104.

Blanchard, P., N. Bancel, G. Boëtsch, Ch. Taraud y D. Thomas 2018. Sexe, Race et Colonies: La Domination des Corps du XVe Siècle à nos Jours. Editorial La Découverte, Paris.

Buikstra, J.E., S.R. Frankenberg y L.W. Konigsberg 1990. Skeletal biological distance studies in American physical anthropology: recent trends. American Journal of Physical Anthropology 82:1-7.

Buikstra, J.E. y D.H. Ubelaker 1994. Standards for Data Collection from Human Skeletal Remains. Arkansas Archaeological Survey Research Series No 44, Arkansas.

Caiaffo, V., P.P.F. Albuquerque, P.V. Albuquerque y B.D.R. Oliveira 2019. Sexual diagnosis through morphometric evaluation of the proximal femur. International Journal of Morphology 37 (2):391-396.

Canals Frau, S. y J. Semper 1956. La Cultura de Agrelo (Mendoza). RUNA, Archivo para las Ciencias del Hombre 2 (2):69-180.

Canals Frau, S. y J. Semper 1957. La Cultura de Agrelo (Mendoza). RUNA, Archivo Para Las Ciencias Del Hombre 7 (2):160-187.

Cardozo, D., H. Lopez, S. Avena y H. Chiavazza 2019. Marcadores Genéticos Mitocondriales de individuos sepultados en el Área Fundacional de la ciudad de Mendoza. Libro de resúmenes XIV Jornadas Nacionales de Antropología Biológica, pp.146. Asociación de Antropología Biológica Argentina, City Bell.

Cavaignac, E., F. Savall, M. Faruch, N. Reina, P. Chiron y N. Telmon 2016. Geometric morphometric analysis reveals sexual dimorphism in the distal femur. Forensic Science International 259:246-e1.

Chiavazza, H. 2005. Los Templos Coloniales como Estructuras Funerarias. Arqueología en la Iglesia Jesuita de Mendoza. British Archaeological Reports, Londres.

Chiavazza, H. 2008. Bases teóricas para el análisis arqueológico de la espacialidad religiosa y los procesos de transformación cultural en la ciudad de Mendoza durante la colonia. Revista de Arqueología Americana 25:225-244.

Chiavazza, H., D. Mansegosa, A. Gámez Mendoza y S. Giannotti 2015a. Funebria católica y estimaciones de sexo y edad en entierros de una ciudad americana colonial (Mendoza siglos XVII-XIX). Revista de Arqueología Histórica Latinoamericana y Argentina 9 (1):35-70.

Chiavazza, H., D. Manegosa y A. Gil. 2015b. Human diet and residential mobility in the Central Western Argentina colony: stable isotopes $(13 \mathrm{C}, 15 \mathrm{~N}, 18 \mathrm{O})$ trends in archaeological bone samples. International Journal of Historical Archaeology 19 (2):289-308.

Chrysostomou, P. y T.J.U. Thompson 2016. Anthropology: Ancestry assessment. Encyclopedia of Forensic and Legal Medicine 1:162-168.

Curry, J. 1984. The Mechanical Adaptation of Bones. Princeton University Press, Princeton. 
Dunn, R., M. Spiros, K. Kamnikar, A. Plemons y J. Heftner 2020. Ancestry estimation in forensic anthropology: A review. WIREs Forensic Science e1369. https://doi.org/10.1002/wfs2.1369

Falconer, D.S. 1986. Introducción a la Genética Cuantitativa. CECSA, México D.F.

Finnegan, M.J. 1978. Non-metric variation of the infracranial skeleton. Journal of Anatomy 125:23-37.

Fitzgerald, C. y S. Hillson 2008. Alternative methods of assessing tooth size in Late Pleistocene and Early Holocene hominids. En Technique and Application in Dental Anthropology, editado por J.D. Irish y G.C. Nelson, pp. 364-388. Cambridge University Press, Cambridge.

Frost, H.M. 2003. Bone's mechanostat: a 2003 update. The Anatomical Record 275A:1081-101.

Giannotti, P.S. 2020a. Resultados preliminares de evaluación de cambios entesiales mediante método Coimbra en poblaciones coloniales españolas de América meridional: ciudad de Mendoza, Argentina (siglos XVII-XIX). Revista Argentina de Antropología Biológica 22 (1):1-19.

Giannotti, P.S. 2020b. El Trabajo durante la Colonia: Análisis Bioarqueológico de Estrés Funcional en Poblaciones Históricas del Norte de Mendoza (Siglos XVI-XIX). Tesis doctoral inédita. Facultad de Filosofía y Letras, Universidad Nacional de Cuyo, Mendoza.

Giannotti, P.S., D.A. Mansegosa y H. Chiavazza 2018. Caries dental y salud oral en poblaciones coloniales de Mendoza (Argentina) durante los siglos XVIII-XIX. Estudios Atacameños. Arqueología y Antropología Surandinas 57:257-276.

Gill, G.W. 2001 Racial variation in the proximal and distal femur: heritability and forensic utility. Journal of Forensic Science 46 (4):791-99.

Gilbert, R. y G.W. Gill 1990. A metric technique for identifying American Indian femora. En Skeletal Attribution of Race, editado por G.W. Gill y S. Rhine, pp. 97-99. Maxwell Museum of Anthropology, University of New Mexico, Albuquerque.

Krenzer, U. 2006. Compendio de Métodos Antropológico Forenses para la Reconstrucción del Perfil Osteobiológico. Centro de Análisis Forense y Ciencias Aplicadas (CAFCA), Serie de Antropología Forense, Guatemala.

Krogman, W. y M. Isçan 1986. The human skeleton in forensic medicine. Segunda Edición. Charles C. Thomas, Springfield.

Larsen, C.S. 1997. Bioarchaeology: Interpreting Behavior from the Human Skeleton. Cambridge University Press, Nueva York.

Larsen, C.S. 2001. Bioarchaeology of Spanish Florida: The Impact of Colonialism. University Press of Florida, Gainesville.

Lieberman, D.E., M.J. Devlin y O.M. Pearson 2001. Articular area responses to mechanical loading: effects of exercise, age, and skeletal location. American Journal of Physical Anthropology 116:266-277.

López Bueis, I. 1998. Indicadores de Presión Ambiental y Dimorfismo Sexual en los Huesos Largos de una Población Española. Tesis doctoral inédita. Facultad Ciencias Biológicas de la Universidad Complutense de Madrid, Madrid.

Lotto, F. y M. Béguelin 2014. Asimetría direccional del postcráneo en poblaciones prehispánicas del sur de Sudamérica. Revista del Museo de Antropología 7 (1):133-142.
Lovejoy, C.O., R.S. Meindl, J.C. Ohman, K.G. Heiple y T.D. White 2002. The Maka femur and its bearing on the antiquity of human walking: Applying contemporary concepts of morphogenesis to the human fossil record. American Journal of Physical Anthropology 119:97- 133.

Luna, L. 2015. Interpretative potential of dental metrics for biodistance analysis in hunter- gatherers from central Argentina. A theoretical-methodological approach. HOMO Journal of Comparative Human Biology 66:432-447.

Mansegosa, D.A. 2015a. Patrones de Variación Morfológica en Poblaciones Históricas del norte Mendocino: Aportes para el Estudio del Proceso de Conquista y Consolidación Colonial. Tesis doctoral inédita. Universidad Nacional de La Plata, La Plata.

Mansegosa, D.A. 2015b.Variación morfológica postcraneal en poblaciones históricas del norte de Mendoza: análisis de la relación entre la estatura y el índice de platimería. Libro de resúmenes de las XXIV Jornadas de Investigación y VI Jornadas de Posgrado, pp. 45. Universidad Nacional de Cuyo, Mendoza.

Mansegosa, D.A. 2016. Estudios sobre salud y enfermedad en poblaciones históricas urbanas de Mendoza (Argentina). Nuevos aportes para el estudio bioarqueológico de La Caridad. Comechingonia virtual Revista de Arqueología 20:111-142.

Mansegosa, D.A., P.S. Giannotti y H. Chiavazza 2017. Aporte de los estudios bioarqueológicos al conocimiento de las poblaciones prehispánicas de Mendoza (Argentina). Revista Española de Antropología Física 38:19-28.

Mansegosa, D.A., P.S. Giannotti, H. Chiavazza y G. Barrientos 2018. Funciones discriminantes para estimar sexo a partir de huesos largos en poblaciones coloniales del centro oeste de Argentina. Chungara Revista de Antropología Chilena 50 (1):155-164.

Mansegosa, D.A. y P.S. Giannotti 2017a. Los nódulos de Schmorl y sus implicancias en la salud de una población histórica colonial (Mendoza, Argentina). Anales de Arqueología y Etnología 72:33-50.

Mansegosa, D.A. y P.S. Giannotti 2017b. Informe antropológico forense de los restos óseos recuperados en la Localidad de Agrelo (Luján de Cuyo, Mendoza), correspondiente al Expediente $\mathrm{N}^{\circ}$ : 57233/19. Manuscrito inédito. Presentado ante el Ministerio Público Fiscal, Mendoza.

Mansegosa, D.A. y P. S. Giannotti 2019. Informe antropológico forense de los restos óseos recuperados en el departamento de Godoy Cruz (Mendoza), correspondiente al Expediente $\mathrm{N}^{\circ}$ : 755914/19. Manuscrito inédito. Presentado ante el Ministerio Público Fiscal, Mendoza.

Mansegosa, D.A, H. Chiavazza y P.S. Giannotti 2018. Estrés metabólico y entierros secundarios: nuevas evidencias para el estudio de las poblaciones coloniales del centro-oeste argentino. Revista Argentina de Antropología Biológica 20 (2):1-18.

Martin, R. y K. Saller 1957. Lehrbuch der Anthropologie Band 1. Gustav Fischer Verlag, Stutgart.

Martínez-Abadías, N., R. González-José, A. González-Martín, S. Van der Molen, A. Talavera, P. Hernández y M. Hernández 2006. Phenotypic evolution of human craniofacial morphology after admixture: a geometric morphometrics approach. American Journal of Physical Anthropology 129:387-398. 
Mazza, B. y M. Béguelin 2013. Determinación sexual de los entierros secundarios del sitio arqueológico Cerro Lutz mediante funciones discriminantes de huesos largos. Cuadernos del Instituto Nacional de Antropología y Pensamiento Latinoamericano del Congreso Internacional de la Cuenca del Plata, pp.121-134.

Miller, M.J. 1995. Femoral platymeria in the Northwestern Plains: genetic and environmental influences. Presented at the $53 \mathrm{rd}$ Annual Plains Anthropologist Conference, Laramie, Wyoming.

Novacheck, T. 1998. The biomechanics of running. Gait and Posture 7:77-95.

Pietrusewsky, M. 2008. Metric analysis of skeletal remains: Methods and applications. En Biological Anthropology of the Human Skeleton, editado por M.A. Katzenberg y S.R. Saunders, pp. 487-532. Segunda Edición. Wiley-Liss, New York.

Pilloud, M.A. y J.T. Hefner 2016. Biological distance Analysis: Forensic and Bioarchaeological Perspectives. Elsevier Inc. Londres.

Prieto, M.R. 1995. Matrimonio y relaciones interétnicas en Mendoza. Ideales y realidad. 1770-1810. Anales de Arqueología y Etnología 50-51:169-186.

Prieto, M.R. 2000 [1983]. Formación y consolidación de una sociedad en un área marginal del Reino de Chile: la Provincia de Cuyo en el siglo XVII. Anales del Instituto de Arqueología y Etnología 52-53:18-366.

Rockhold, L.A. 1998. Secular Change in External Femoral Measures from 1840 to 1970: A Biomechanical Interpretation. MSc thesis, University of Tennessee, Knoxville.

Ross, A.H., D.E. Slice, D.H. Ubelaker y A.B. Falsetti 2004. Population affinities of 19th century Cuban crania: implications for identification criteria in South Florida Cuban Americans. Journal of Forensic Science 49:1-6.

Ruff, C.B. 1995. Biomechanics of the hip and birth in early. American Journal of Physical Anthropology 98:527-574.

Ruff, C.B. 1987. Sexual dimorphism in human lower limb bone structure: re-lationship to subsistence strategy and sexual dimorphism. Journal of Human Evolution 16:391-416.

Ruff, C.B. 1999. Skeletal structure and behavioral patterns of prehistoric Great Basin populations. En Prehistoric Lifeways in the Great Basin Wetlands: Bioarchaeological Reconstruction and Interpretation, editado por B. E. Hemphill y C. S. Larsen, pp. 290-320. University of Utah Press, Salt Lake City.

Ruff, C.B y B.M. Auerbach 2004. Human body mass estimation: a comparison of "morphometric" and "mechanical" methods. American Journal of Physical Anthropology 125 (4):331-342.

Ruff, C.B., C.S. Larsen y W.C Hayes 1984. Structural changes in the femur with the transition to agriculture in the Georgia coast. American Journal of Physical Anthropology 64:125-136.

Rusconi, C. 1962. Poblaciones Pre y Posthispánicas de Mendoza. Gobierno de Mendoza, Mendoza.

Schobinger, J. 1969-1970. Un osario prehistórico en Chacras de Coria (Mendoza). Anales de Arqueología y Etnología 24-25:229-237.

Scheuer, L. y S. Black 2004. The Juvenil Skeleton. Elsevier. Academic Press. Londres.

Trotter, M. 1970. Estimation of stature from intact long limb bones. Personal Identification in Mass Disasters, editado por T.D. Stewart, pp 71-83. Smithsonian Press, Washington DC.

Tallman, S.D. y A.P. Winburn 2015. Forensic applicability of femur subtrochanteric shape to ancestry assessment in Thai and white American males. Journal of Forensic Sciences 60 (5):1283-1289.

Verdaguer, J. 1931. Historia Eclesiástica de Cuyo. Tipografía Saleciana, Milano. Tomo I y II. Mendoza.

Wescott, D.J. 2001. Structural Variation in the Humerus and Femur in the American Great Plains and Adjacent Regions: Differences in Subsistence Strategy and Physical Terrain. PhD dissertation, University of Tennessee, Knoxville.

Wescott, D.J. 2005. Population variation in femur subtrochanteric shape. Journal of Forensic Science 50 (2):286-293.

Wescott, D.J. 2006. Ontogeny of femur subtrochanteric shape in Native Americans and American blacks and whites. Journal of Forensic Sciences 51 (6):1240-1245.

Wescott, D.J. y D. Srikanta 2008. Testing assumptions of the Gilbert and Gill method for assessing ancestry using the femur subtrochanteric shape. HOMO: Journal of Comparative Human Biology 59 (5):347-363.

Wescott, D.J. y L.R. Zephro 2016. Secular changes in the femur diaphyseal biomechanical properties of American whites. Human Biology 88 (1):38-46.

Zuluaga, R.M. 1964. El Cabildo de la Ciudad de Mendoza. Su primer medio siglo de existencia. Instituto de Historia. Mendoza.

Zuluaga, R.M. 1970. La Trata de Negros en la Región Cuyana durante el Siglo XVII. Revista de la Junta de Estudios Históricos de Mendoza, Segunda Época, 6 (I). 